\title{
Vertebrate hepatic lipase genes and proteins: a review supported by bioinformatic studies
}

This article was published in the following Dove Press journal:

Open Access Bioinformatics

22 April 2011

Number of times this article has been viewed

\section{Roger S Holmes ${ }^{1,2}$ \\ John L VandeBerg' \\ Laura A Cox'}

'Department of Genetics, Southwest National Primate Research Center,

Texas Biomedical Research Institute, San Antonio, Texas, USA; ${ }^{2}$ School

of Biomolecular and Physical

Sciences, Griffith University, Nathan,

Queensland, Australia
Correspondence: Roger S Holmes Department of Genetics, Southwest National Primate Research Center,

Texas Biomedical Research Institute,

San Antonio, TX 78227, USA

$\mathrm{Tel}+12102589687$

Fax + I 2102589600

Email rholmes@sfbrgenetics.org
Abstract: Hepatic lipase (gene: $L I P C$; enzyme: HL; E.C.3.1.1.3) is one of three members of the triglyceride lipase family that contributes to vascular lipoprotein degradation and serves a dual role in triglyceride hydrolysis and in facilitating receptor-mediated lipoprotein uptake into the liver. Amino acid sequences, protein structures, and gene locations for vertebrate LIPC (or Lipc for mouse and rat) genes and proteins were sourced from previous reports and vertebrate genome databases. Lipc was distinct from other neutral lipase genes (Lipg encoding endothelial lipase and $L p l$ encoding lipoprotein lipase [LPL]) and was located on mouse chromosome 9 with nine coding exons on the negative strand. Exon 9 of human LIPC and mouse and rat Lipc genes contained "stop codons" in different positions, causing changes in C-termini length. Vertebrate HL protein subunits shared 58\%-97\% sequence identities, including active, signal peptide, disulfide bond, and N-glycosylation sites, as well as proprotein convertase ("hinge") and heparin binding regions. Predicted secondary and tertiary structures revealed similarities with the three-dimensional structure reported for horse and human pancreatic lipases. Potential sites for regulating $L I P C$ gene expression included $\mathrm{CpG}$ islands near the 5"-untranslated regions of the mouse and rat LIPC genes. Phylogenetic analyses examined the relationships and potential evolutionary origins of the vertebrate $L I P C$ gene family with other neutral triglyceride lipase gene families ( $L I P G$ and $L P L)$. We conclude that the triglyceride lipase ancestral gene for vertebrate neutral lipase genes $(L I P C, L I P G$, and $L P L)$ predated the appearance of fish during vertebrate evolution.

Keywords: vertebrates, amino acid sequence, hepatic lipase, evolution, gene duplication

\section{Introduction}

Hepatic lipase (HL; gene $L I P C$; E.C.3.1.1.3) is one of three members of the triglyceride lipase family that contributes to lipoprotein degradation within the circulation system. ${ }^{1-3}$ HL also regulates the metabolism of low-density lipoprotein, intermediate-density lipoprotein, and high-density lipoprotein particles and is capable of catalyzing the hydrolysis of phospholipids, triglycerides, and acyl-CoA thioesters. ${ }^{4,5}$ Endothelial lipase (EL; gene LIPG; E.C.3.1.1.3) is a related family member that plays a major role in high-density lipoprotein cholesterol metabolism in the body, catalyzing phospholipase and triglyceride lipase activities ${ }^{6-8}$ and lipoprotein lipase (LPL; gene $L P L$; E.C.3.1.1.34) functions in the hydrolysis of triglycerides of circulating chylomicrons and very low-density lipoproteins. ${ }^{9-11}$ These enzymes share sequence similarities $(38 \%-44 \%$ identities) and are usually referred to as the vascular lipase gene family ${ }^{7,12,13}$ because of their contributions to plasma lipoprotein, cholesterol, and triglyceride phenotypes and to the development of coronary heart diseases in human and animal populations. ${ }^{14-21}$ 
The human LIPC gene is located on chromosome 15 and comprises $158.3 \mathrm{~kb}$ nucleotides on the direct strand with nine exons and eight introns and encodes a 449 amino acid protein subunit. ${ }^{3,22}$ Genetic variants have been described that cause HL deficiency and associated hyperlipidemia. ${ }^{23}$ Several promoter polymorphisms in linkage disequilibrium have also been identified, and the more frequent $-250 \mathrm{G}>\mathrm{A}$ substitution in the LIPC promoter region is associated with changes in plasma lipid concentrations and the risk of coronary artery disease in some ethnic groups. ${ }^{24}$ LIPC is expressed predominantly in the liver, where the enzyme contributes significantly to the determination of lipoprotein levels, structure, and metabolism. ${ }^{1-3}$ Studies of Lipc $^{-} /$Lipc $^{-}$knockout mice have supported multiple roles for HL in vascular lipoprotein metabolism, including a lipolytic role and a ligand binding function facilitating lipoprotein uptake, which influence lipoprotein particle size in the circulation. ${ }^{17}$ Following synthesis in the liver endoplasmic reticulum, rat HL is processed by the hydrolysis of the N-terminal leader peptide and acquisition of oligosaccharides within the Golgi and is then rapidly secreted and subsequently bound to heparin sulfate proteoglycans on the surface of hepatocytes. ${ }^{25} \mathrm{HL}$ forms a dimeric subunit structure ${ }^{26}$ exhibiting similarities with EL, which behaves as a homodimer with a proposed head-to-tail conformation, ${ }^{27}$ and is subject to proprotein convertase cleavage at a site in the "hinge" region separating the N- and C-terminal enzyme domains. ${ }^{28}$ Three-dimensional studies of a related mammalian lipase (LIPP, pancreatic lipase) $)^{29,30}$ have enabled identification of three major structural domains for the mammalian neutral lipase family, including an $\mathrm{N}$-terminal domain with a catalytic triad of serine, aspartate, and histidine residues; a "lid" domain that covers the active site and contributes to the specificity for triglyceride and phosphoglyceride substrates; and a C-terminal or "plat" domain, which contributes to lipid binding and specificity. ${ }^{31,32}$

This paper examines and reviews the gene structures and amino acid sequences for several vertebrate $L I P C$ genes and proteins; the predicted secondary and tertiary structures for vertebrate HL enzymes; and the structural, phylogenetic, and evolutionary relationships for these genes and enzymes with those for human and mouse lipase neutral lipase gene families, $L I P G$ (encoding endothelial lipase), and $L P L$ (encoding lipoprotein lipase).

\section{Methods}

\section{Vertebrate LIPC gene and HL identification}

Protein BLAST (Basic Local Alignment Search Tool) analyses generated several vertebrate HL amino acid sequences from the National Center for Biotechnology
Information (NCBI) database (http://blast.ncbi.nlm.nih. gov/Blast.cgi) ${ }^{33}$ (Table 1). Nonredundant protein sequence databases for vertebrate genomes were examined using the blastp algorithm, including human (Homo sapiens), ${ }^{34}$ chimpanzee (Pan troglodytes), ${ }^{35}$ orangutan (Pongo abelii) (http://genome.wustl.edu), rhesus monkey (Macaca mulatta), ${ }^{36}$ cow (Bos taurus) (http://hgsc.bcm.tmc.edu/ projects/bovine), mouse (Mus musculus), ${ }^{37}$ rat (Rattus norvegicus), ${ }^{38}$ rabbit (Oryctolagus cuniculus) (http://www. broadinstitute.org/science/projects/mammals-models/rabbit/ rabbit-genome-sequencing-project), opossum (Monodelphis domestica), ${ }^{39}$ chicken (Gallus gallus), ${ }^{40}$ frog (Xenopus tropicalis) (http://genome.jgi-psf.org/Xentr3/Xentr3.home. html), and zebrafish (Danio rerio) (http://www.sanger. ac.uk/Projects/D_rerio/). Predicted or previously reported vertebrate HL-like protein sequences were then subjected to analyses of protein and gene structures (Table 1).

BLAT (BLAST-like Alignment Tool) analyses were subsequently undertaken for each of the predicted HL amino acid sequences using the University of California, Santa Cruz genome browser (http://genome.ucsc.edu/cgi-bin/hgBlat) ${ }^{41}$ with the default settings to obtain the predicted locations for each of the mammalian $L I P C$ genes, including predicted exon boundary locations and gene sizes. BLAT analyses were also undertaken for human $L P L$ (encoding lipoprotein lipase) ${ }^{9}$ and $L I P G$ (encoding endothelial lipase) ${ }^{6-8}$ (see Table 1). Structures for human, mouse, and rat isoforms (splicing variants) were obtained using the AceView website to examine predicted gene and protein structures ${ }^{42}$ (http://www.ncbi.nlm. nih.gov/IEB/Research/Acembly/index.html?human).

\section{Predicted structures and properties of vertebrate hepatic lipases}

Secondary and tertiary structures for human and other vertebrate HL-like proteins were predicted using Web tools from PSIPRED v2.5 (http://bioinf.cs.ucl.ac.uk/psipred/) and SWISS MODEL (http://swissmodel.expasy.org), respectively. ${ }^{43,44}$ The structure for the human pancreatic lipase-colipase complex ${ }^{45}$ served as a reference for the predicted horse LIPP (pancreatic lipase) structure (previously reported by Bourne et $\mathrm{al}^{30}$ ) and the human, opossum and zebrafish LIPC tertiary structures, with modeling ranges of residues $18-465,25-471,4-448$ and 25-485, respectively. Theoretical isoelectric points and molecular weights (http://au.expasy.org/tools/pi_tool.html), location of signal peptide cleavage sites (http://www.cbs.dtu. $\mathrm{dk} /$ services/SignalP/) ${ }^{46}$ and potential N-glycosylation sites (http://www.cbs.dtu.dk/services/NetNGlyc/) for vertebrate LIPC proteins were obtained using Web tools. 


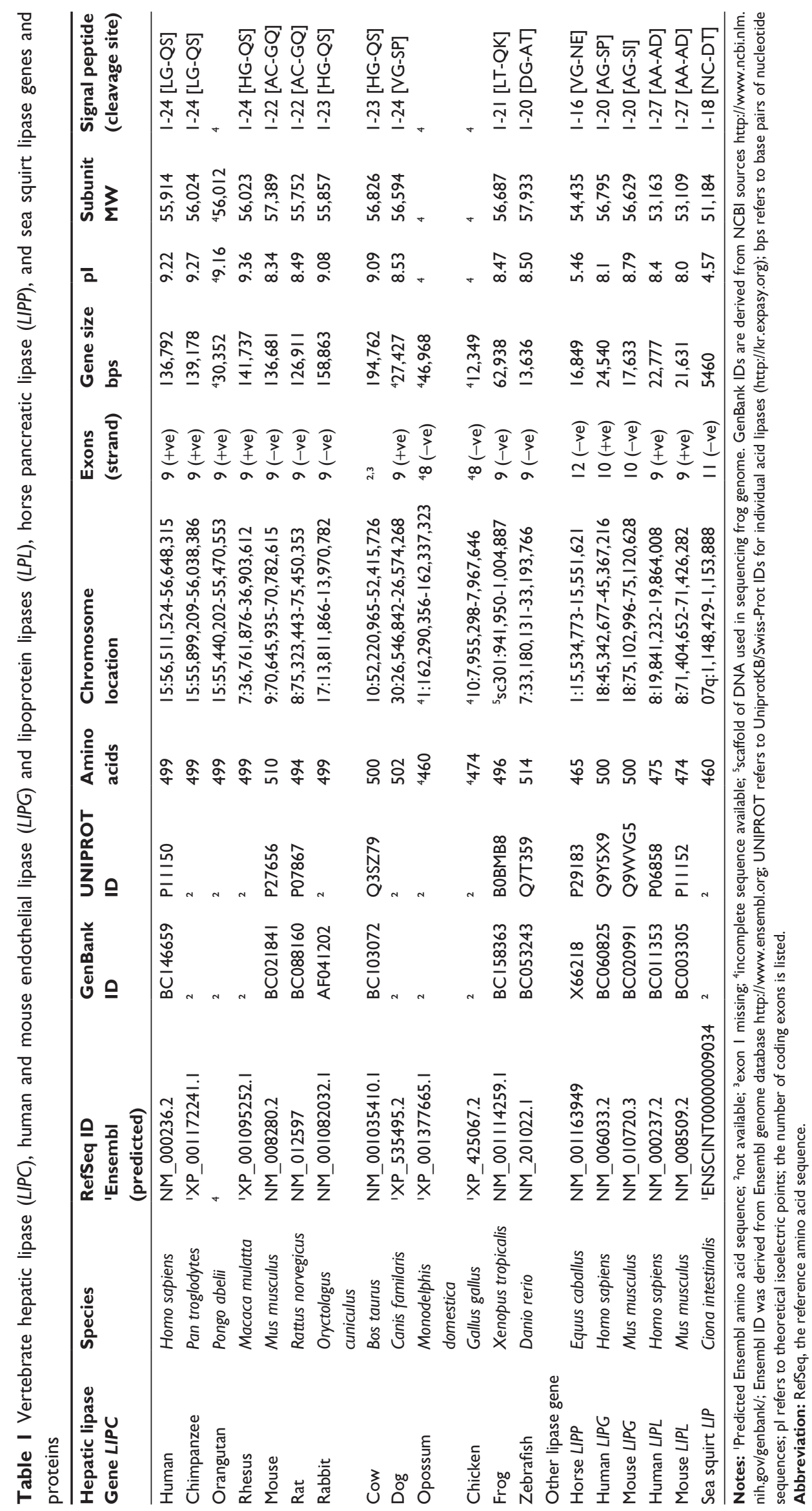




\section{Phylogenetic studies and sequence divergence}

Alignments of vertebrate HL with human and mouse EL and LPL sequences were assembled using BioEdit v.5.0.1 and the default settings. ${ }^{47}$ Alignment ambiguous regions, including the amino and carboxyl termini, were excluded prior to phylogenetic analysis, yielding alignments of 395 residues for comparisons of vertebrate HL, human and mouse EL, and LPL sequences with the sea squirt (Ciona intestinalis) lipase sequence (Table 1). Evolutionary distances were calculated using the Kimura option ${ }^{48}$ in TREECON. ${ }^{49}$ Phylogenetic trees were constructed from evolutionary distances using the neighbor-joining method ${ }^{50}$ and rooted with the sea squirt lipase sequence. Tree topology was re-examined by the bootstrap method (100 bootstraps were applied) of resampling and only values that were highly significant $(\geq 90)$ are shown. ${ }^{51}$

\section{Results and discussion}

\section{Alignments of vertebrate $\mathrm{HL}$ amino acid sequences}

The deduced amino acid sequences for dog, frog, and zebrafish HL are shown in Figure 1 together with previously reported sequences for human $\mathrm{HL},{ }^{3,22}$ mouse $\mathrm{HL},{ }^{52}$ rat $\mathrm{HL},{ }^{53,54}$ and horse pancreatic lipase (LIPP) ${ }^{30}$ (Table 1). Alignments of human and other vertebrate HL sequences examined showed between $49 \%$ and $98 \%$ identities, suggesting that they are products of the same family of genes, whereas comparisons of sequence identities of vertebrate HL proteins with human and mouse EL and LPL and horse LIPP exhibited lower levels of sequence identities, EL (38\% and 42\%, respectively), LPL (44\% and 45\%, respectively), and LIPP (25\%), indicating that they are members of distinct but related neutral lipase families (Table 2).

The amino acid sequences for human, chimp, orangutan, rhesus monkey, and rabbit HL contained 499 residues whereas mouse, rat, cow, dog, and frog HL contained 510, 494, 500, 502, and 496 amino acids, respectively (Table 1; Figure 1). Previous three-dimensional studies of horse pancreatic lipase $(\text { LIPP })^{30}$ and modeling studies of human $\mathrm{EL}^{29}$ have enabled predictions of key residues for vertebrate HL amino acid sequences (numbers refer to human HL). These included the catalytic triad for the active site (Ser168, Asp194, and His279); the hydrophobic N-terminus signal peptides (see also Table 1), which facilitate enzyme secretion into the

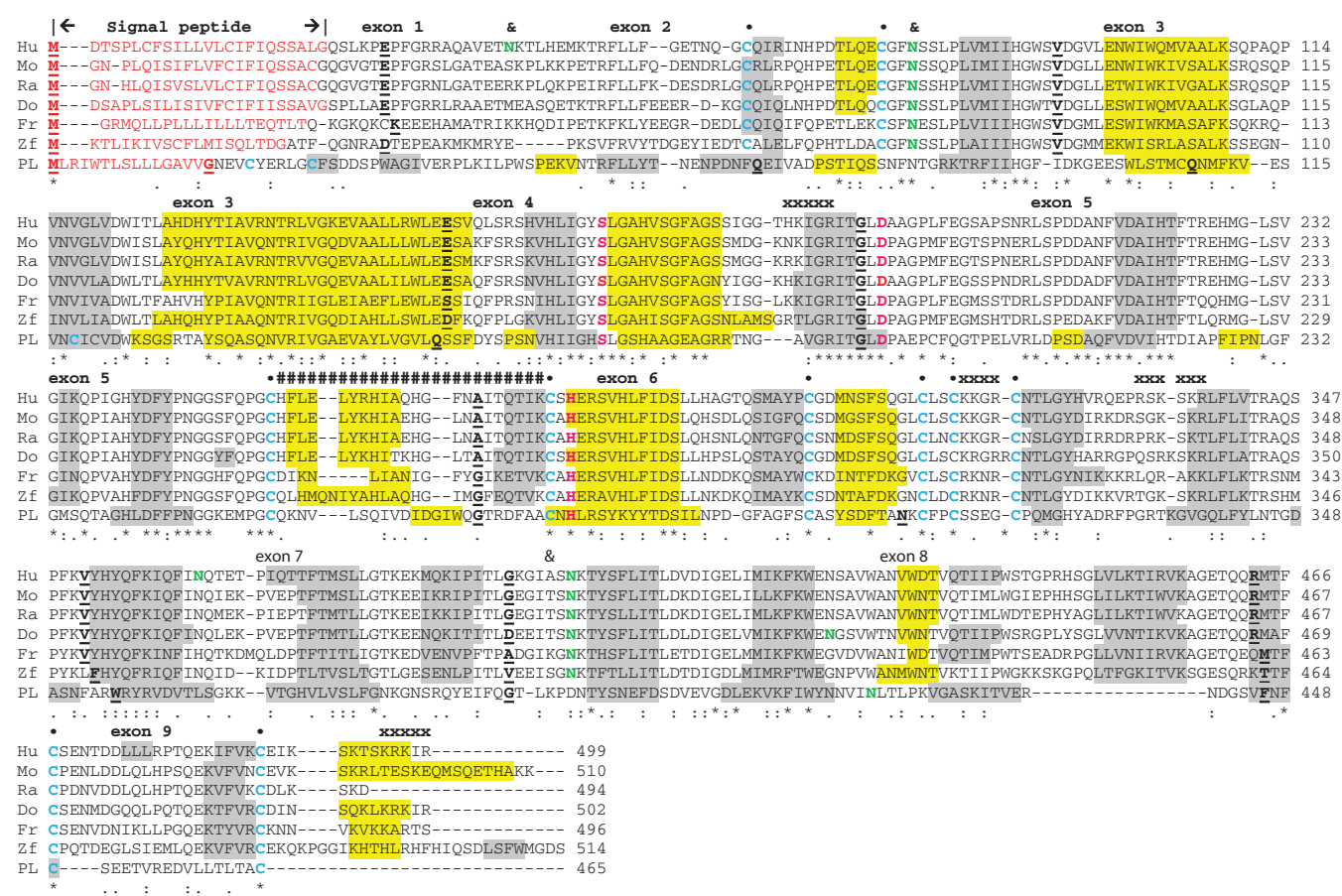

Figure I Amino acid sequence alignments for vertebrate hepatic lipase (HL) and horse pancreatic lipase (LIPP) sequences. See Table I for sources of HL and horse LIPP sequences.

Notes: *shows identical residues for lipase subunits; similar alternative residues; dissimilar alternative residues; residues involved in $\mathrm{N}$-signal peptide formation are shown in red; $\mathrm{N}$-glycosylated (marked as and for human $\mathrm{HL}$ ) and potential $\mathrm{N}$-glycosylated Asn sites are in green bold; active site triad residues Ser (S), Asp (D), and His (H) are in pink bold; predicted disulfide bond Cys residues are shown in blue bold (•); $\alpha$-helix for horse LIPP or predicted for vertebrate HL is in shaded yellow; $\beta$-sheet for horse LIPP or predicted for vertebrate HL is in shaded grey; bold underlined font shows residues corresponding to known or predicted exon start sites; exon numbers refer to human LIPC gene exons; \#\# refers to residues that correspond to the horse LIPP "lid" region; xxxxx refers to the four predicted "heparin binding" regions for human HL. Abbreviations: Do, dog HL; Fr, frog HL; Hu, human HL; Mo, mouse HL; PL, horse pancreatic lipase; Ra, rat HL; Zf, zebrafish HL. 


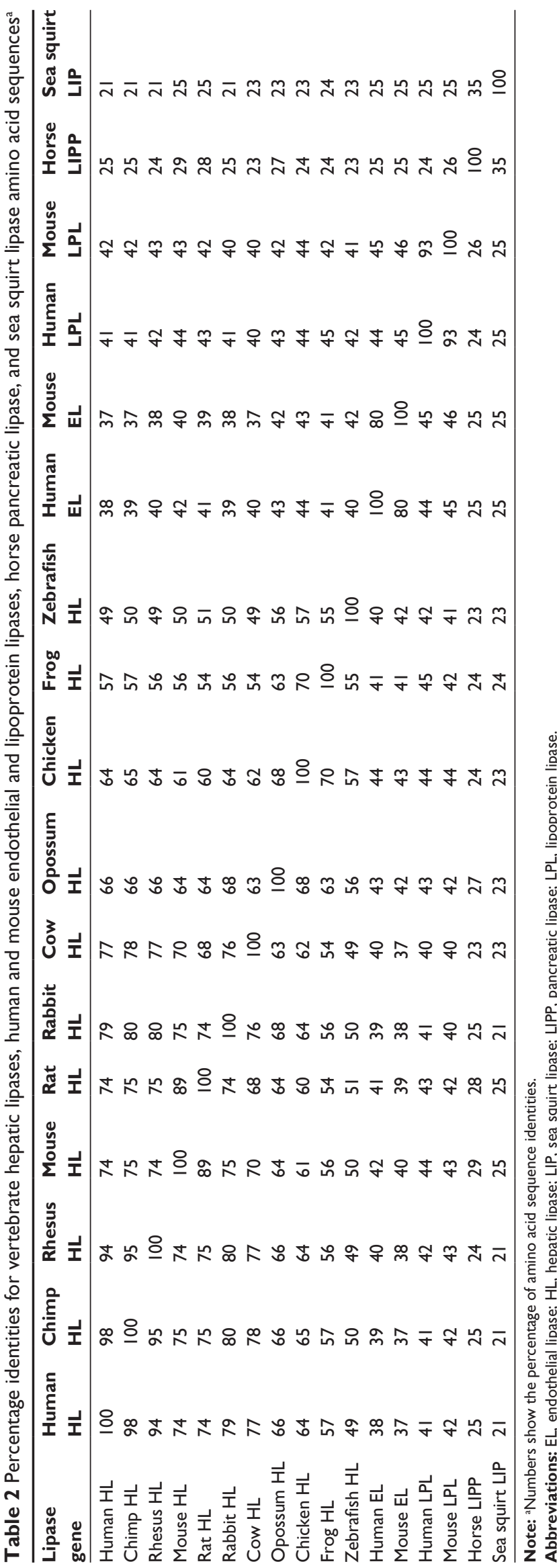

circulation system; ${ }^{25}$ five disulfide bond-forming residues (Cys62/Cys75, Cys254/Cys277, Cys302/Cys313, Cys316/ Cys321, and Cys467/Cys487); the predicted "lid" region (255-276), which covers the active site and participates in lipid substrate binding in analogous lipases; ${ }^{31,32}$ and a predicted "hinge" region for vertebrate HL (332Arg-333Ser334Lys-335Ser) (based on sequence similarity with human EL [327Arg-328Asn-329Lys-330Arg], which contains a proprotein convertase proteolytic cleavage site). ${ }^{29-32}$ With the exception of the $\mathrm{N}$-terminus signal peptides, the vertebrate HL sequences were strictly conserved or underwent conservative substitutions, which may reflect the essential nature of these residues in contributing to HL structure and function. The N-terminal region (residues 1-63) underwent major changes in the number and sequence of amino acid residues but retained a predicted signal peptide property in each case (Figure 1; Table 1). The horse LIPP sequence shared the catalytic triad residues, four of the five disulfide bonds predicted for the vertebrate HL sequences, and an $\mathrm{N}$-signal peptide sequence property; however, other sequences were distinct with only $25 \%$ identical residues observed for horse LIPP and human HL.

Figure 2 compares vertebrate HL sequences for four putative heparin binding sites described for human HL, which contain clusters of basic amino acid residues with different consensus sequences. ${ }^{56-59}$ These sites are apparently responsible for HL binding to heparin sulfate proteoglycans on the surface of parenchymal cell microvilli where the enzyme functions in liver lipoprotein catabolism. ${ }^{58,59}$ Several vertebrate HL sequences have also been compared with human LPL and EL putative heparin binding sites, as well as those for human apolipoproteins APOB and APOE, the major proteins of chylomicrons, low-density lipoprotein, and very low-density lipoprotein, which function as recognition signals for the cellular binding and internalization of lowdensity lipoprotein particles. ${ }^{60}$ Several differences from heparin binding consensus sequences were observed. For consensus sequence 1 (XBBBXXBX where B refers to a basic amino acid and $X$ to any other amino acid), human, rhesus, and mouse HL and human LPL sequences lacked the first basic amino acid, and human EL contained only two of four basic amino acids. Consensus sequence 2 (XBBXBX) showed consistency for all vertebrate HL, human LPL and EL, and APOB sequences examined with the exception of rabbit HL, which lacked one of the B residues, and dog HL, which contained an extra B residue. Several differences were observed for consensus sequence 3 (XBXBBX), including rat and dog HL and human EL and LPL. The C-terminal 
Gene Protein Vertebrate

LIPC HL

Frog

LPL LPL Human

LIPG EL Human

$A P O B$ ApoB Human

APOE APOE Human

\begin{tabular}{|c|c|c|c|}
\hline \multicolumn{4}{|c|}{ Consensus Heparin Binding Sequences } \\
\hline 1 & 2 & 3 & 4 (c-terminal) \\
\hline XBBBXXBX & XBBXB-X & $\mathrm{X}-\mathrm{BXBBX}$ & ВХВXXВBВXB \\
\hline 3GTHKIGRI & 316 CKKGR-C & 334 S-KSKRL & 489KSKTSKRKIR \\
\hline GTRKIGRI & 316 CKKGR-C & 334 S-KNKKL & 489KSKTSKRQIR \\
\hline GTNKIGRI & 317CKKGR-C & 335 G-KSKRL & 490KSKRLTESKEQMSQETHAKK \\
\hline AGRKIGRI & 317CKKGR-C & 335 R-KSKTL & 490 KSKD \\
\hline 3 GKHKIGRI & 316CTKGR-C & $334 \mathbf{S}-\mathbf{K G K R L}$ & 489NPKKLKLKIK \\
\hline 4 GKHKIGRI & 317CKRGRRC & 338 SRKSKRL & 492NSQKLKRKIR \\
\hline 4 GTNKIGRI & 277 CKKGR-C & $295 Q-K S K K L$ & 450GSKPQNQRLR \\
\hline GTNKIGRI & 292 CRKNR-C & 310 P-KSRKL & 46 6RLRQHERK \\
\hline GLKKIGRI & 312 CRKNR-C & 330 Q-RAKKL & 486NVKVKKARTS \\
\hline 4TNKKVNRI & 305 CRKNR-C & $323 \overline{\mathbf{K}}-\mathbf{R S S K M}$ & 467 KSLNKKSG \\
\hline 4 VKGTVGRI & 311CRKNR-C & 32 9K-RNSKM & 485GWRMKNETSPTVELP \\
\hline TRKRGLKL & 113 LKKTK-N & $2145 \mathbf{T}-\mathbf{K K Y R I}$ & \\
\hline
\end{tabular}

Figure 2 Comparative amino acid sequences for predicted heparin binding sequences for vertebrate hepatic lipase (HL) and human lipoprotein lipase (LPL), endothelial lipase (EL), apolipoprotein B (APOB), and apolipoprotein E (APOE) sequences. Four predicted heparin binding sites are shown based on previous studies ${ }^{56-60}$ and the predicted vertebrate $H L$ sequences reported in this paper.

Abbreviations: $\mathrm{B}$, basic amino acid; $\mathrm{K}$, lysine; $\mathrm{R}$, arginine; $\mathrm{X}$, any other amino acid residue.

heparin binding site (consensus sequence 4) showed major differences among the HL sequences examined, especially for mouse and rat HL, which lacked this motif. This may explain why mouse HL is predominantly found in the circulation system as compared with human HL, which is released into the circulation following heparin administration. ${ }^{61}$

Four N-glycosylation sites have previously been reported for human HL at 42Asn-43Lys-44Thr, 78Asn-70Ser-71Ser, 362Asn-363Gln-364Thr, and 397Asn-398Lys-399Thr. ${ }^{62,63}$ A comparative analysis of potential $\mathrm{N}$-glycosylation sites for vertebrate HL has shown that there are seven sites overall, although only two of these have been predominantly retained for the 13 vertebrate HL sequences examined (designated as sites 3 and 7) (Table 3). Site-directed mutagenesis studies of site 3 (human HL 78Asn) have demonstrated that this $\mathrm{N}$-glycosylation site is required for the efficient secretion of this liver enzyme. ${ }^{64,65}$

\section{Predicted secondary and tertiary structures for vertebrate hepatic lipases}

Predicted secondary structures for vertebrate HL sequences were compared with the previously reported secondary structure for horse LIPP (pancreatic lipase) ${ }^{30}$ (Figure 1). $\alpha$-Helix and $\beta$-sheet structures for the vertebrate HL protein sequences were examined and found to be similar for several regions with the horse LIPP secondary structures. Consistent structures were predicted near key residues or functional domains, including the $\beta$-sheet and $\alpha$-helix structures near

Table 3 Predicted $\mathrm{N}$-glycosylation sites for vertebrate hepatic lipases. Numbers refer to amino acids in the acid sequences, including $\mathrm{N}$-asparagine, K-lysine, I-isoleucine, M-methionine, H-histidine, S-serine, R-arginine, T-threonine, Q-glutamine, and V-valine. Note that seven potential sites were identified, including four confirmed sites for human LIPC (HL) (sites I, 3, 5, and 7). High- (yellow highlighted) and lower probability $\mathrm{N}$-glycosylation sites were identified using the NetNGlyc I.0 Web server (http://www.cbs.dtu.dk/services/NetNGlyc/)

\begin{tabular}{|c|c|c|c|c|c|c|c|c|c|}
\hline Vertebrate & Species & Site I & Site 2 & Site 3 & Site 4 & Site 5 & Site 6 & Site 7 & No. of sites \\
\hline Human & Homo sapiens & $42 \mathrm{NKT}$ & & $78 N S S$ & & $362 \mathrm{NQT}$ & & 397NKT & 4 \\
\hline Chimpanzee & Pan troglodytes & $42 \mathrm{NKT}$ & & $78 N S S$ & & $362 \mathrm{NQT}$ & & $397 \mathrm{NKT}$ & 4 \\
\hline Orangutan & Pongo abelii & $42 \mathrm{NKT}$ & & $78 N S S$ & & 362NQT & & & 3 \\
\hline Rhesus & Macaca mulatta & $42 \mathrm{NKT}$ & & $78 N S S$ & & 362NQT & & $397 \mathrm{NKT}$ & 4 \\
\hline Mouse & Mus musculus & & & 79NSS & & & & $398 \mathrm{NKT}$ & 2 \\
\hline Rat & Rattus norvegicus & & & 79NSS & & & & $398 \mathrm{NKT}$ & 2 \\
\hline Rabbit & Oryctolagus cuniculus & & & 78NSS & & & & 397NKT & 2 \\
\hline Cow & Bos taurus & & 67NHS & $78 N S S$ & & 363NQT & & 398NET & 4 \\
\hline Dog & Canis familaris & & & 79NSS & & & & $400 \mathrm{NKT}$ & 2 \\
\hline Opossum & Monodelphis domestica & $39 N S S$ & & & I43NGT & $358 \mathrm{NKT}$ & $378 N F T$ & & 4 \\
\hline Chicken & Gallus gallus & & $55 \mathrm{NAS}$ & & & & $374 N K T$ & & 2 \\
\hline Frog & Xenopus tropicalis & & & 78NES & & & & $394 \mathrm{NKT}$ & 2 \\
\hline Zebrafish & Danio rerio & & & $75 N S S$ & & & & $395 \mathrm{NKT}$ & 2 \\
\hline
\end{tabular}




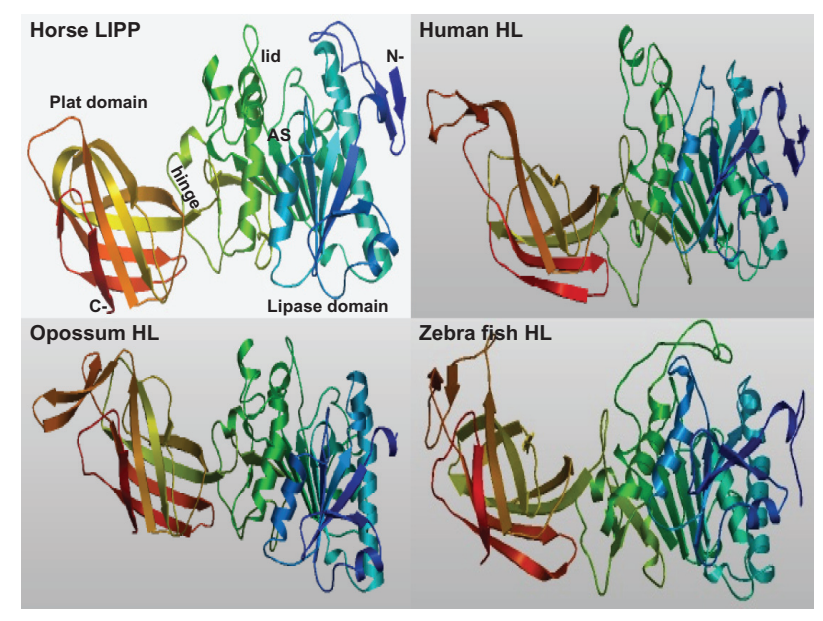

Figure 3 Predicted Tertiary Structures for Horse LIPP and for Human, Opossum and Zebrafish LIPC Predicted horse LIPP and human, opossum and zebrafish LIPC tertiary structures were obtained using SWISS MODEL methods; the rainbow color code describes the tertiary structures from the $\mathrm{N}$ - (blue) to C-termini (red color); the horse LIPP tertiary structure shows the $\mathrm{N}$ - and C-termini, the 'lipase', 'lid' (in yellow) and 'plat' domains which are separated by a 'hinge' region; and the active site region (AS) for horse LIPP is identified ( based on the horse LIPP structure reported by Bourne et al. ${ }^{30}$ ).

the active site residues (human HL numbers used) Ser168, Asp 194, and His279; the "lid" domain (residues 255-276); and the "hinge" region, which commences with an $\alpha$-helix and concludes with a $\beta$-sheet (residues 333-339). Figure 3 describes predicted tertiary structures for human, opossum, and zebrafish HL protein sequences and shows significant similarities for these polypeptides with horse pancreatic lipase (LIPP). ${ }^{30}$ The three LIPP and HL domains were readily apparent, including the N-terminal "lipase" domain with the active site triad residues buried under the "lid" domain observed for horse LIPP. The "lid" has previously been shown to contribute to the preference for triglyceride and phopholipid substrates of vascular lipases HL and LPL..$^{31,65}$ A "hinge" region was also observed for these vertebrate HL proteins, separating the "lipase" and "plat" domains, with the latter having a "sandwich-like" $\beta$-pleated sheet structure. The "plat" domain for HL and LPL has been shown to be essential for binding these enzymes to lipoprotein micelles and also contributes to preferences in lipoprotein binding. ${ }^{29}$ These comparative studies for other vertebrate HL proteins suggest that these properties and key sequences are substantially retained for all of the vertebrate sequences examined.

\section{Predicted gene locations and exonic structures for vertebrate LIPC genes}

Table 1 summarizes the predicted locations for vertebrate LIPC genes based on BLAT interrogations of several vertebrate genomes using the reported sequences for human, ${ }^{6,7}$ mouse, ${ }^{66}$ and rat $\mathrm{HL}^{38}$ and the University of California, Santa Cruz genome browser. ${ }^{41}$ The predicted primate $L I P C$ genes were transcribed on the positive strand, whereas other vertebrate $L I P C$ genes were transcribed on the negative strand. Figure 1 summarizes the predicted exonic start sites for vertebrate $L I P C$ genes with each having nine coding exons, in identical or similar positions to those predicted for the human $L I P C$ gene. ${ }^{67}$
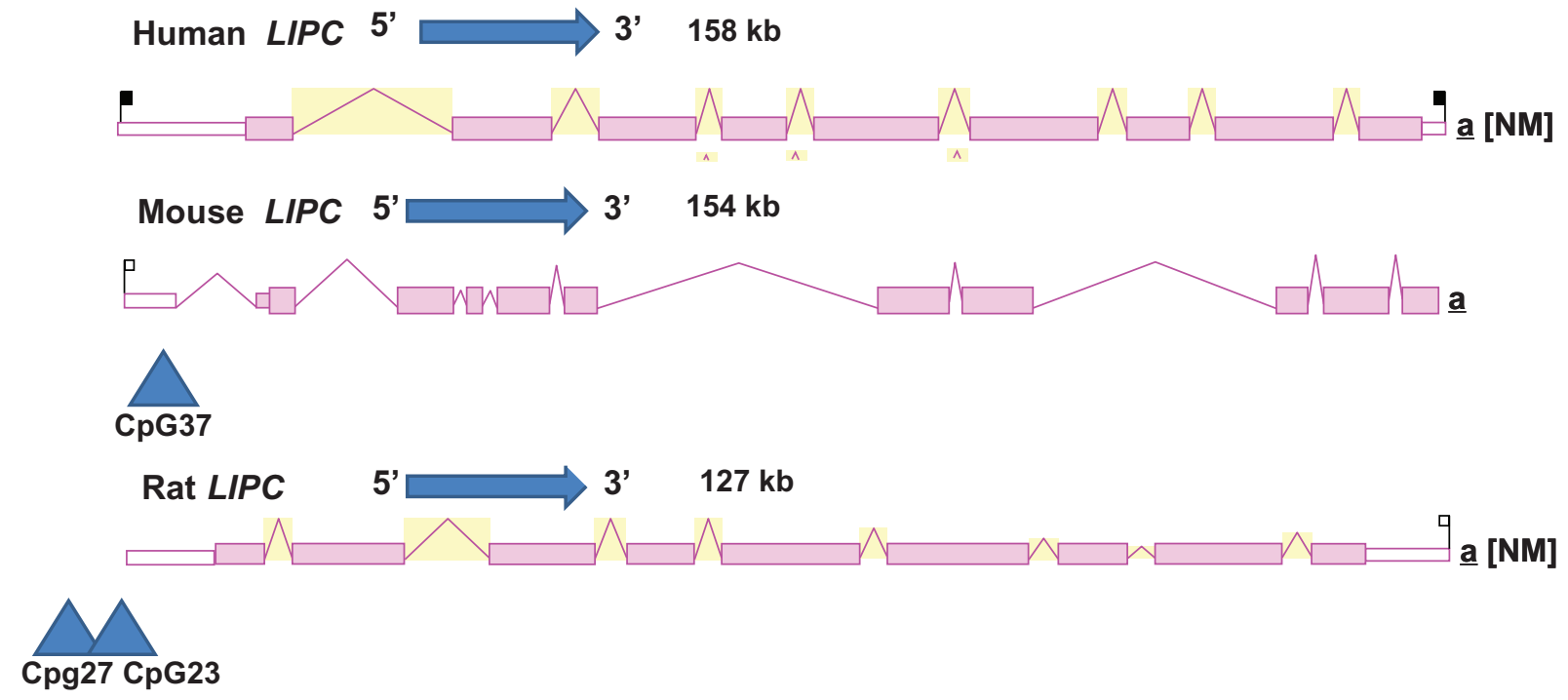

Figure 4 Gene structures and major splicing variant for the human, mouse, and rat LIPC transcripts. Derived from the AceView website ${ }^{42}$

http://www.ncbi.nlm.nih.gov/IEB/Research/Acembly/. Mature isoform variants (a) are shown with capped 5"- and 3"- ends for the predicted mRNA sequences; NM refers to the NCBI reference sequence; exons are in pink; the directions for transcription are shown as 5" $\rightarrow 3$ "; blue triangles show predicted CpG island sites at or near the 5 "untranslated regions of the gene; sizes of mRNA sequences are shown in kilobases (kb). 


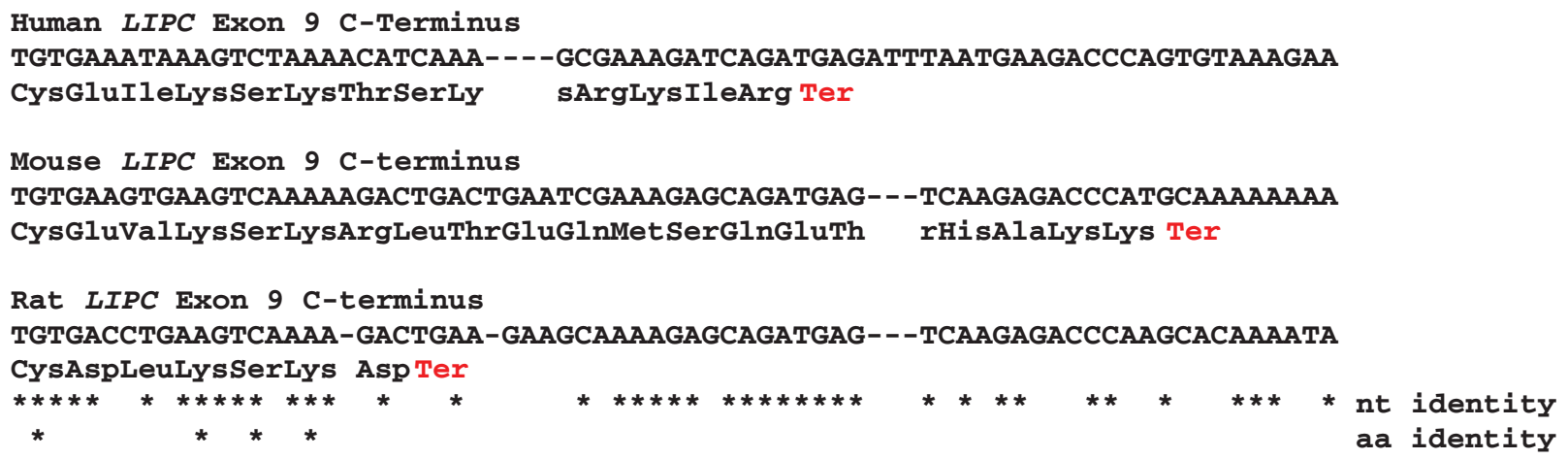

Figure 5 Nucleotide and amino acid sequence alignments for human, mouse, and rat LIPC genes and hepatic lipase proteins: predicted c-termini and exon 9 sequences. Identical nucleotide (nt) and amino acid (aa) sequences are shown (*). Ter (in red) refers to predicted terminating codons.

Figure 4 illustrates the predicted structures of mRNA for human, mouse, and rat $L I P C$ transcripts for the major transcript isoform in each case. ${ }^{42}$ The transcripts were $127-158$ kbs in length with nine introns present for these LIPC mRNA transcripts. Figure 5 examines the predicted amino acid and nucleotide sequence for the $\mathrm{C}$-terminus end of exon 9 human, mouse, and rat $L I P C$ sequences. It is proposed that exon 9 has undergone nucleotide substitutions or deletions/insertions that have introduced a termination codon for the rat LIPC gene encoding an incomplete $\mathrm{C}$-terminus for rat $\mathrm{HL}$ and an extended C-terminus for mouse HL. The significance of these differences in rodent LIPC structure has been previously discussed in terms of the observed changes in HL binding to heparin sulfate proteoglycans on liver parenchymal cells where the enzyme functions in liver lipoprotein catabolism. . $^{57}$

\section{Phylogeny and divergence of hepatic lipase and other vertebrate lipase sequences}

A phylogenetic tree (Figure 6) was calculated by the progressive alignment of 12 vertebrate LIPC amino acid sequences with human and mouse LIPL and LIPG sequences that was "rooted" with the Ciona intestinalis (sea squirt) lipase

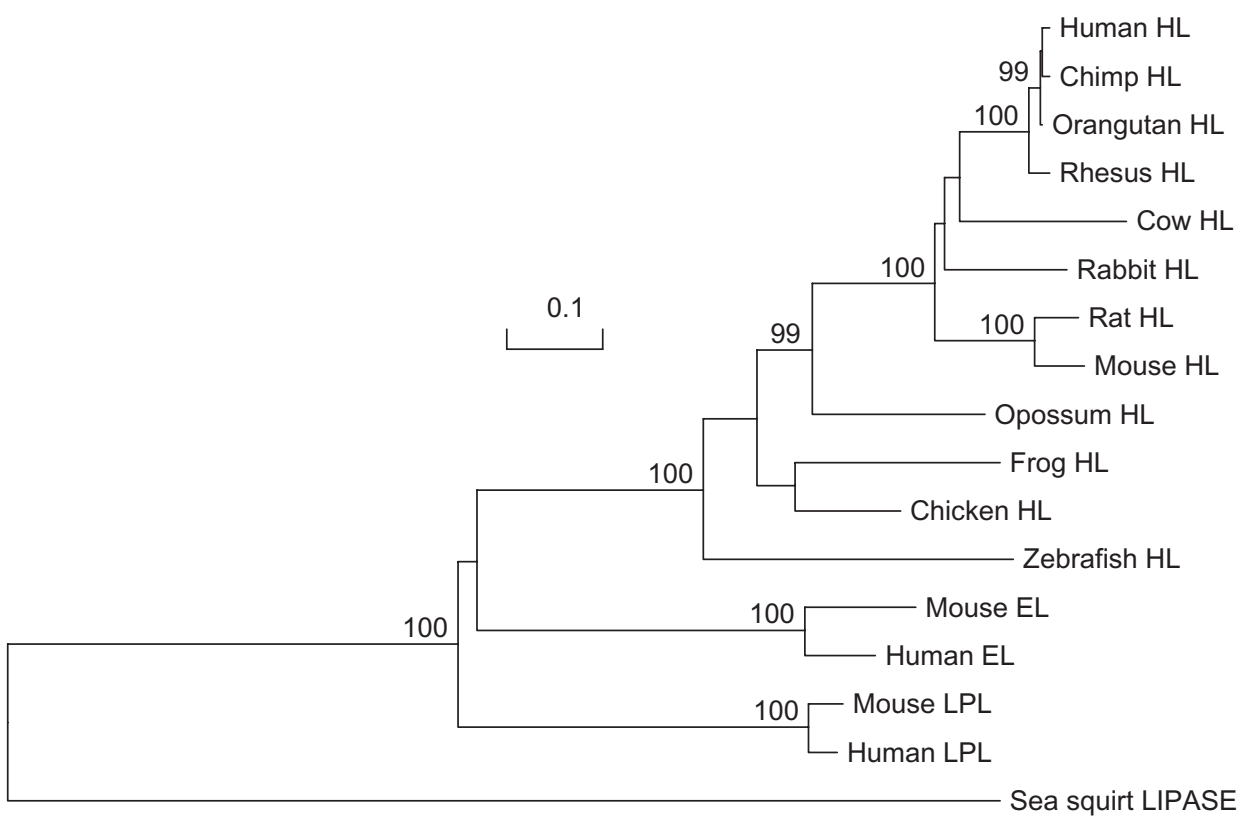

Figure 6 Phylogenetic tree of vertebrate hepatic lipase (HL), human and mouse lipoprotein lipase (LPL) and endothelial lipase (EL), and sea squirt lipase amino acid sequences. The tree is labeled with the lipase name and the name of the animal and is "rooted" with the Ciona intestinalis (sea squirt) lipase sequence. Note the major cluster of vertebrate $\mathrm{HL}$ sequences, which is distinct from the human and mouse LPL and EL and the sea squirt lipase sequences. A genetic distance scale is shown. The number of times a clade (sequences common to a node or branch) occurred in the bootstrap replicates are shown. Only replicate values of 90 or more that are highly significant are shown, with 100 bootstrap replicates performed in each case. Note the significant separation of clades for the three human vascular lipases (LPL, EL, and HL). 
sequence (see Table 1). The phylogram showed clustering of the LIPC sequences into groups that were consistent with their evolutionary relatedness as well as distinct groups for human and mouse LIPL and LIPG sequences, which were distinct from the sea squirt lipase sequence. These groups were significantly different from each other (with bootstrap values of $\sim 100 / 100)$. It is apparent from this study of vertebrate LIPC genes and proteins that this is an ancient protein for which a proposed common ancestor for the LIPC, LIPG, and $L I P L$ neutral lipase genes may have predated the appearance of bony fish, which occurred $>500$ million years ago. ${ }^{68}$ This proposal is consistent with a previous report from Cohen, ${ }^{69}$ which described predicted amino acid sequences for human and pufferfish (Takifugu rubripes) LIPG, LIPL, and LIPC.

\section{Conclusion}

These results indicate that vertebrate LIPC genes and encoded HL enzymes represent a distinct gene and enzyme family of neutral lipases that share key conserved sequences that have been reported for other neutral lipases previously studied. ${ }^{6-11}$ This enzyme has a distinct property among the neutral lipases studied in being the major liver lipase and playing a major role in the catabolism of lipoproteins in the circulation system. ${ }^{1-3}$ HL is encoded by a single gene for the vertebrate genomes studied and usually contains nine coding exons. The rat LIPC gene encoded a shorter form of this enzyme (494 residues compared with 499 amino acids for most mammalian HL sequences) due to the presence of a termination codon located in exon 9. Predicted secondary structures and tertiary structures for vertebrate HL proteins showed a strong similarity with human and horse pancreatic lipases (LIPP). ${ }^{29,30}$ Three major structural domains were apparent for vertebrate HL, including the "lipase" domain containing the catalytic triad residues; the "lid", which covers the active site and may contribute to the substrate specificities of neutral lipases; ;1,64 and the "plat" domain, which contributes to lipoprotein binding. ${ }^{65}$ Phylogenetic studies using amino acid sequences for 13 vertebrate HL lipases, human and mouse LPL and EL, and an invertebrate lipase indicated that the LIPC gene has appeared early in vertebrate evolution, probably prior to the appearance of bony fish more that 500 million years ago.

\section{Acknowledgments}

This project was supported by National Institutes of Health Grants P01 HL028972 and P51 RR013986. In addition, this investigation was conducted in facilities constructed with support from Research Facilities Improvement Program Grant Numbers 1 C06 RR13556, 1 C06 RR15456, and 1 C06 RR017515. We gratefully acknowledge Dr Bharet Patel from Griffith University for his assistance with the phylogenetic analyses.

\section{Disclosure}

The authors report no conflicts of interest in this work.

\section{References}

1. Martin GA, Busch SJ, Meredith GD, et al. Isolation and cDNA sequence of human postheparin plasma hepatic triglyceride lipase. J Biol Chem. 1988;263:10907-10914.

2. Datta S, Luo CC, Li WH, et al. Human hepatic lipase. Cloned cDNA sequence, restriction fragment length polymorphisms, chromosomal localization, and evolutionary relationships with lipoprotein lipase and pancreatic lipase. J Biol Chem. 1988;263:1107-1110.

3. Cai SJ, Wong DM, Chen SH, Chan L. Structure of the human hepatic triglyceride lipase gene. Biochem. 1989;28:8966-8971.

4. Zambon A, Austin MA, Brown BG, et al. Effect of hepatic lipase on LDL in normal men and those with coronary artery disease. Arterioscler Thromb. 1993;13:147-153.

5. Santamarina-Fojo S, Haudenschild C, Amar M. The role of hepatic lipase in lipoprotein metabolism and atherosclerosis. Curr Opin Lipidol. 1998;9:211-219.

6. Jaye M, Lynch KJ, Krawiec J, Marchadier D, et al. A novel endothelialderived lipase that modulates HDL metabolism. Nature Genetics. 1999; 21:424-428.

7. Hirata K, Dichek HL, Cioffi JA, et al. Cloning of a unique lipase from endothelial cells extends the lipase gene family. J Biol Chem. 1999;274: 14170-14175.

8. Holmes RS, VandeBerg JL, Cox LA. Vertebrate endothelial lipase: comparative studies of an ancient gene and protein in vertebrate evolution. Genetica. 2011; DOI 10.1007/s10709-011-9549-1.

9. Wion KL, Kirchgessner TG, Lusis AJ, et al. Human lipoprotein lipase complementary DNA sequence. Science. 1987;235:1638-1641.

10. Dichek HL, Fojo SS, Beg OU, et al. Identification of two separate allelic mutations in the lipoprotein lipase gene of a patient with the familial hyperchylomicronemia syndrome. J Biol Chem. 1991;266: 473-477.

11. Benlian P, De Gennes JL, Foubert L, et al. Premature atherosclerosis in patients with familial chylomicronemia caused by mutations in the lipoprotein lipase gene. New Eng J Med. 1996;335:848-854.

12. Ma K, Cilingiroglu M, Otvos JD, et al. Endothelial lipase is a major genetic determinant for high-density lipoprotein concentration, structure and metabolism. Proc Natl Acad Sci U S A. 2003;100:2748-2753.

13. Brown RJ, Rader DJ. Lipases as modulators of atherosclerosis in murine models. Curr Drug Targets. 2007;8:1307-1319.

14. Guerra RJ, Wang S, Grundy M, Cohen JC. A hepatic lipase (LIPC) allele associated with high plasma concentrations of high density lipoprotein cholesterol. Proc Natl Acad Sci U S A. 1997;94:4532-4537.

15. Murtomaki, S, Tahvanainen E, Antikainen M, et al. Hepatic lipase gene polymorphisms influence plasma HDL levels. Results from Finnish EARS: participants of the European Atherosclerosis Research study. Arterioscler Thromb Vasc Biol. 1997;17:1877-1879.

16. de Andrade FM, Silveira FR, Arsand M, et al. Association between -250G/A polymorphism of the hepatic lipase gene promoter and coronary artery disease and HDL-C levels in a southern Brazilian population. Clin Genet. 2004;65:390-395.

17. Gonzalez-Navarro H, Nong Z, Amar MJA, et al. The ligand-binding function of hepatic lipase modulates the development of atherosclerosis in transgenic mice. J Biol Chem. 2004;279:45312-45321. 
18. Eller P, Schgoer W, Mueller T, et al. Hepatic lipase polymorphism and increased risk of peripheral arterial disease. J Intern Med. 2005;258: 344-348.

19. Chasman DI, Paré G, Mora S, et al. Forty-three loci associated with plasma lipoprotein size, concentration, and cholesterol content in genome-wide analysis. PLoS Genet. 2009;5:e1000730.

20. Hodoglugil U, Williamson DW, Mahley RW. Polymorphisms in the hepatic lipase gene affect plasma HDL-cholesterol levels in a Turkish population. J Lipid Res. 2010;51:422-430.

21. Baylin A, Ruiz-Narvaez E, Jensen MK, et al. Association between hepatic lipase $-514 \mathrm{C} / \mathrm{T}$ promoter polymorphism and myocardial infarction is modified by history of hypercholesterolemia and waist circumference. Nutr Metab Cardiovasc Dis. 2010;20:498-504.

22. Ameis D, Stahnke G, Kobayashi J, et al. Isolation and characterization of the human hepatic lipase gene. J Biol Chem. 1990;265:6552-6555.

23. Hegele RA, Tu L, Connelly PW. Human hepatic lipase mutations and polymorphisms. Hum Mutat. 1992;1:320-321.

24. Meng L, Ruixing Y, Yiyang L, et al. Association of LIPC $-250 \mathrm{G}>\mathrm{A}$ polymorphism and several environmental factors with serum lipid levels in the Guangxi Bai Ku Yao and Han populations. Lipids Health Dis. 2010;9:28.

25. Cisar LA, Bensadoun A. Characterization of the intracellular processing and secretion of hepatic lipase in FU5AH rat hepatoma cells. Biochim Biophys Acta. 1987;927:305-314.

26. Hill JS, Davis RC, Yang D, et al. Human hepatic lipase subunit structure determination. J Biol Chem. 1996;271:22931-22936.

27. Griffon N, Jin W, Petty TJ, et al. Identification of the active form of endothelial lipase, a homodimer in a head-to-tail conformation. $J$ Biol Chem. 2009;284:23322-23330.

28. Jin W, Fuki IV, Seidah NG, et al. Proprotein convertases are responsible for proteolysis and inactivation of endothelial lipase. J Biol Chem. 2005; 280:36551-36559.

29. Winkler FK, D'Arcy A, Hunziker W. Structure of human pancreatic lipase. Nature. 1990;343:771-774.

30. Bourne Y, Martinez C, Kerfelec B, et al. Horse pancreatic lipase. The crystal structure refined at 2.3-A resolution. J Mol Biol. 1994;238: 709-732.

31. Dugi KA, Dichek HL, Santamarina-Fojo S. Human hepatic and lipoprotein lipase: the loop covering the catalytic site mediates lipase substrate specificity. J Biol Chem. 1995;270:25396-25401.

32. Broedl UC, Jin W, Fuki IV, Glick JM, Rader DJ. Structural basis for endothelial lipase tropism for HDL. FASEB J. 2004;18:1891-1893.

33. Altschul F, Vyas V, Cornfield A, et al. Basic local alignment search tool. J Mol Biol. 1990;215:403-410.

34. International Human Genome Sequencing Consortium. Initial sequencing and analysis of the human genome. Nature. 2001;409: 860-921.

35. Chimpanzee Sequencing and Analysis Consortium. Initial sequence of the chimpanzee genome and comparison with the human genome. Nature. 2005;437:69-87.

36. Rhesus Macaque Genome Sequencing and Analysis Consortium. Evolutionary and biomedical insights from the rhesus macaque genome. Science. 2007;316:222-234.

37. Mouse Genome Sequencing Consortium. Initial sequencing and comparative analysis of the mouse genome. Nature. 2002;420: $520-562$.

38. Rat Genome Sequencing Project Consortium. Genome sequence of the brown Norway rat yields insights into mammalian evolution. Nature. 2004;428:493-521.

39. Mikkelsen TS, Wakefield MJ, Aken B, et al. Genome of the marsupial Monodelphis domestica reveals innovation in noncoding sequences. Nature. 2007;447:167-177.

40. International Chicken Genome Sequencing Consortium. Sequence and comparative analysis of the chicken genome provide unique perspectives on vertebrate evolution. Nature. 2004;432:695-716.

41. Kent WJ, Sugnet CW, Furey TS, et al. The human genome browser at UCSC. Genome Res. 2003;12:994-1006.
42. Thierry-Mieg D, Thierry-Mieg J. AceView: a comprehensive cDNA-supported gene and transcripts annotation. Genome Biol. 2006;7:S12. http:// www.ncbi.nlm.nih.gov/IEB/Research/Acembly/index.html?human.

43. McGuffin LJ, Bryson K, Jones DT. The PSIPRED protein structure prediction server. Bioinformatics. 2000;16:404-405.

44. Kopp J, Schwede T. The SWISS-MODEL repository of annotated three-dimensional protein structure homology models. Nucleic Acids Res. 2004;32:D230-D234.

45. Egloff MP, Marguet F, Buono G, et al. The $2.46 \AA$ \& resolution structure of the pancreatic lipase-colipase complex inhibited by a C11 alkyl phosphonate. Biochemistry. 1995;34:2751-2762.

46. Emmanuelsson O, Brunak S, von Heijne G, Nielson H. Locating proteins in the cell using TargetP, SignalP and related tools. Nat Protocols. 2007; 2:953-971.

47. Kimura M. The Neutral Theory of Molecular Evolution. Cambridge: Cambridge University Press; 1983.

48. Van De PeerY, de Wachter R. TreeCon for Windows: a software package for the construction and drawing of evolutionary trees for the Microsoft Windows environment. Comput Appl Sci. 1994;10:569-570.

49. Saitou N, Nei M. The neighbour-joining method: a new method for reconstructing phylogenetic trees. Mol Biol Evol. 1987;4:406-425.

50. Hall TA. BioEdit: a user-friendly biological sequence alignment editor and analysis program for Windows 95/98/NT. Nucleic Acids Symp Ser. 1999;41:95-98.

51. Felsenstein J. Confidence limits on phylogenies: an approach using the bootstrap. Evolution. 1985;39:783-791.

52. Chang SF, Netter HJ, Will H. Characterization of cDNA encoding the mouse hepatic triglyceride lipase and expression by in vitro translation. FEBS Lett. 1991;289:69-72.

53. Komaromy MC, Schotz MC. Cloning of rat hepatic lipase cDNA: evidence for a lipase gene family. Proc Natl Acad Sci U S A. 1987;84: $1526-1530$.

54. Sensel MG, Legrand-Lorans A, Wang ME, Bensadoun A. Isolation and characterization of clones for the rat hepatic lipase gene upstream regulatory region. Biochim Biophys Acta. 1990;1048:297-302.

55. Stahnke G, Sprengel R, Augustin J, Will J. Human hepatic triglyceride lipase: cDNA cloning, amino acid sequence and expression in a cultured cell line. Differentiation. 1987;35:45-52.

56. Sendak RA, Berryman DE, Gellman G, et al. Binding of hepatic lipase to heparin, Identification of specific-heparin-binding residues in two distinct positive clusters. J Lipid Res. 2000;41:260-268.

57. Perret B, Mabile L, Martinez L, et al. Hepetic lipase: structure/ function relationship, synthesis, and regulation. J Lipid Res. 2002;43: 1163-1169.

58. Sanan DA, Fan J, Bensadoun A, Taylor JM. Hepatic lipase is abundant on both hepatocyte and endothelial cell surfaces in the liver. J Lipid Res. 1997;38:1002-1013.

59. Breedveld B, Schoonderwoerd AJ, Verhoeven R, et al. Hepatic lipase is localized at the parenchymal cell microvilli in rat liver. Biochem. 1997;321:425-430.

60. Boerwinkle E, Brown SA, Rohrbach K, et al. Role of apolipoprotein $\mathrm{E}$ and $\mathrm{B}$ gene variation in determining response of lipid, lipoprotein, and apolipoprotein levels to increased dietary cholesterol. Am J Hum Genet. 1991;49:1145-1154.

61. Peterson J, Bengtsson-Olivecrona G, Olivecrona T. Mouse preheparin plasma contains high levels of hepatic lipase with low affinity for heparin. Biochim Biophys Acta. 1986;878:65-70.

62. Wolle J, Jansen H, Smith LC, Chan L. Functional role of N-linked glycosylation in human hepatic lipase: asparagine-56 is important for both enzyme activity and secretion. $J$ Lipid Res. 1993;34:2169-2176.

63. Ben-Zeev O, Stahnke G, Liu RC, et al. Lipoprotein lipase and hepatic lipase: the role of asparagines linked glycosylation in the expression of a functional enzyme. J Lipid Res. 1994;35:1511-1523.

64. Kobayashi J, Applebaum-Bowden D, Dugi KA, et al. Analysis of protein structure-function in vivo. Adenovirus-mediated transfer of lipase lid mutants in hepatic lipase-deficient mice. J Biol Chem. 1996;271: 26296-26301. 
65. Wong H, Davis RC, Nikazy J, et al. Domain exchange: characterization of a chimeric lipase of hepatic lipase and lipoprotein lipase. Proc Natl Acad Sci U S A. 1991;88:11290-11294.

66. The MGC Project Team. The status, quality, and expansion of the NIH full-length cDNA project: the Mammalian Gene Collection (MGC). Genome Res. 2004;14:2121-2127.

67. Clark HF, Gurney AL, Abaya E, et al. The secreted protein discovery initiative (SPDI), a large-scale effort to identify novel human secreted and transmembrane proteins: a bioinformatics assessment. Genome Res. 2003;13:2260-2264.
68. Donoghue PCJ, Benton MJ. Rocks and clocks: calibrating the tree of life using fossils and molecules. Trends Genet. 2007;22: 424-431.

69. Cohen JC. Endothelial lipase: direct evidence for a role in HDL metabolism. J Clin Invest. 2003;111:318-321.

Open Access Bioinformatics

\section{Publish your work in this journal}

Open Access Bioinformatics is an international, peer-reviewed, open access journal publishing original research, reports, reviews and commentaries on all areas of bioinformatics. The manuscript management system is completely online and includes a very quick and fair
Dovepress

peer-review system. Visit http://www.dovepress.com/testimonials.php to read real quotes from published authors. 